\title{
La educación geográfica y el riesgo de desastres en las escuelas municipales del área urbana de Constitución, Chile
}

\author{
Geography Education and the Risk of Disasters in Public City \\ Schools in Constitucion, Chile
}

Ana Isabel Huaico-Malhue*

\begin{abstract}
RESUMEN
El aprendizaje de las características de los fenómenos naturales, tales como terremotos, tsunamis e inundaciones, forma parte del currículo nacional de la asignatura de Historia, Geografía y Ciencias Sociales. En este contexto, el siguiente trabajo investigó la relación entre la ocurrencia de desastres y el conocimiento geográfico relacionado con riesgos y desastres naturales en el segundo ciclo de la enseñanza básica en el área urbana de Constitución en Chile. Se aplicaron encuestas y test de conocimientos, con bajos porcentajes de aprobación en casi todos los contenidos evaluados, por lo que se concluye que, a pesar de los acontecimientos catastróficos acontecidos en los últimos años en la ciudad de Constitución, el nivel de conocimiento en la materia es bajo, por lo que se hace necesario un programa de educación para la gestión del riesgo de desastres a nivel escolar.
\end{abstract} Palabras clave:

\begin{abstract}
Learning about the characteristics of natural phenomena, like earthquakes, tsunamis and flooding, is part of the national curriculum for History, Geography and Social Sciences. Within this context, the following paper studies the relationship between the occurrence of disasters and geographic knowledge related to
\end{abstract} educación, geografía, riesgos, desastres.

Keywords: education, geography, risks, disasters.

Chilena, Doctora en Medio Ambiente y Desarrollo, Universidad de Baja California, México. Académica del Departamento de Geografía, Facultad de Arquitectura, Geografía y Urbanismo, Universidad de Concepción, Chile. E-mail: ahuaico@udec.cl El desarrollo de este trabajo de investigación contó con la colaboración de Javiera Gutiérrez Vergara, Andrés Lara Ibarra, Fabián Puente Yáñez y Gabriel Muñoz Pizarro, licenciados en educación de la Universidad Autónoma de Chile. 
risks and natural disasters in the second cycle of elementary education in city schools of Constitucion, Chile. Surveys and tests of knowledge were applied, showing low performance in nearly all of the contents evaluated, leading to the conclusion that despite catastrophic events that have occurred during the last few years in the city of Constitucion, the level of knowledge on the topic is low, and an education program on disaster risk management is needed in schools. 
La enseñanza de la geografía en Chile está unida con la de otras dos disciplinas, como la historia y las ciencias sociales, estas últimas ligadas al espacio geográfico, ya que es ahí donde ocurren los acontecimientos históricos. Lo anterior tiene sentido en un país cuya diversidad geográfica lo hace no tan solamente un territorio de grandes contrastes, sino también un país que presenta recurrencia de diferentes fenómenos naturales, que históricamente han afectado de diferentes formas al territorio nacional. En este contexto, la educación formal se convierte en una vía para formar ciudadanos informados, comprometidos y actores de cambio social, en temas tales como medioambiente, riesgos naturales y cambio climático.

La educación ambiental es una herramienta social que permite a los individuos lograr un conocimiento significativo del entorno habitado, por lo que dichos saberes deben estar ligados a las ciencias naturales, pero también a la geografía (Ordoñez-Díaz et al., 2017: 2). Por consiguiente, el siguiente trabajo investiga la relación entre la ocurrencia de desastres, el conocimiento geográfico y los riesgos naturales en estudiantes del segundo ciclo de la enseñanza básica, en cuatro establecimientos municipales localizados en el área urbana del municipio de Constitución en la Séptima Región del Maule en Chile.

Es necesario mencionar que la ciudad de Constitución fue una de las más afectadas por el terremoto y tsunami de 2010, así como también la más impactada por los incendios forestales de 2017. De esta forma, se estudia el nivel de instrucción de los estudiantes en tópicos relacionados con el conocimiento geográfico, ya que, como menciona Arenas,

"el posicionamiento de la geografía como disciplina científica, contribuye a la alfabetización científica de los habitantes de un país o región. Esta alfabetización busca generar personas capaces de entender los fenómenos desde una perspectiva geográfica, que contribuya a una apropiación de las problemáticas del territorio en el cual se desarrollan, en otras palabras, se promueve una alfabetización geográfica, que es el propósito fundamental de la educación geográfica (2013: 144)".

En este sentido, es necesario mencionar que las definiciones de "riesgo" y de "desastre" han evolucionado a través de los años (Re- 
yes et al., 2017). Al respecto, los textos escolares hacen mención a los conceptos de "riesgo natural" y "desastre natural". Briones-Gamboa alude a que la idea de "riesgo" tiene dos connotaciones básicas: riesgo natural y riesgo social. El primero, según el autor, se liga al daño eventual y potencial destructivo de los fenómenos ambientales, mientras que el segundo a los procesos sociales que generan riesgo (2007: 9). Otro aspecto es que el riesgo es definido como la suma de la amenaza o cálculo de la probabilidad de ocurrencia de un fenómeno natural potencialmente peligroso, con la vulnerabilidad o exposición del proyecto y de las personas a ese evento natural adverso (Camus et al., 2017: 7).

Petit-Breuilh define y entiende que:

"los desastres en general han sido definidos como fenómenos que afectan directamente a las personas y/o sectores productivos, y que provocando daños de consideración en la infraestructura físicas y de servicios, empeorando las condiciones de vida de diversos sectores de la población alterando su actividad cotidiana; por este motivo, se suele denominar desastres naturales a aquellos sucesos catastróficos relacionados de alguna manera con procesos naturales (2004: 23)".

Molina et al. definen y ejemplifican que:

"los desastres naturales son característicos en Chile, terremotos, tsunamis, erupciones volcánicas e inundaciones son fenómenos relativamente frecuentes, cuya acción muchas veces altera la forma como los grupos humanos se relacionan con la naturaleza. Esto, debido a su capacidad de modificar prácticas culturales, sociales y económicas, reorientando así, el devenir de un determinado territorio, tal como sucedió con el terremoto $9,5^{\circ}$ Richter y posterior tsunami de 1960, en la comuna de Saavedra, Chile (2018: 189)".

Uno de los compromisos de los países es reducir el riesgo de desastres. La Estrategia Internacional para la Reducción de Desastres de las Naciones Unidas lo define como la expectativa de posibles pérdidas que ocasionaría un desastre en términos de vidas, condiciones de salud, medios de sustento, bienes y los servicios, y que podrían ocurrir en una comunidad o sociedad particular y en un periodo específico de tiempo en el futuro (UNISDR, 2009). 


\section{Educación geográfica para la reducción del riesgo de desastre}

Alpizar (2009: 34) menciona que "la educación y el desarrollo de conocimientos sobre gestión del riesgo, se convierte en una de las actividades elementales de la reducción de desastres, y son los niños, niñas y jóvenes estudiantes, los verdaderos actores de la gestión prospectiva del riesgo y sus educadores los medios más efectivos para hacer realidad esta intencionalidad". Por esto, en todo el mundo se ha dado relevancia a la educación para el riesgo de desastres, documentándose iniciativas en más de setenta países. Asimismo, se menciona que todavía ningún esfuerzo ha abordado amenazas múltiples, ha sido de alcance nacional o se ha integrado plenamente a los sistemas escolares (Naciones Unidas, 2007).

Según la ISDR (2011), para lograr integrar a la comunidad educativa en de la temática de riesgos y prevención de ellos, se debe promover:

- La inclusión del conocimiento sobre la reducción del riesgo de desastres en los planes de estudio, en todos los niveles.

- El uso de canales formales e informales para llegar a los niños niñas y jóvenes con información sobre reducción del riesgo de desastres.

- La integración de la reducción de riesgo de desastres como un elemento intrínseco en la década de educación para el desarrollo sostenible.

- La integración de programas educativos de reducción de riesgo dirigidos a sectores específicos.

Del mismo modo, la UNESCO (2019) establece que:

"la integración del enfoque de la gestión del riesgo en el sector educativo es determinante para incrementar la concientización sobre el efecto y causa de los desastres. Las escuelas que incluyen acciones de gestión del riesgo contribuyen a una cultura para la prevención, esencial para el desarrollo sostenible de los países. Estas acciones reducen los riesgos de desastres y fortalecen las capacidades de las comunidades más vulnerables para responder a las emergencias".

Uno de los desafíos recurrentes de la educación geográfica es fomentar en los estudiantes la capacidad de pensar críticamente sobre fenómenos geográficos, en un mundo cada vez más globalizado. Este 
desafío ha sido ilustrado de forma cruda por los desastres naturales que han afectado la naturaleza, interconectados con los procesos políticos, económicos y culturales (Alderman, 2002). Por esto, dentro del currículo nacional, la enseñanza de la geografía necesita de modificaciones que promuevan un aprendizaje centrado en el pensamiento y razonamiento espacial, y que además fusione, dentro de su investigación, aspectos teóricos y prácticos (Bednarz, 2004), de modo que los estudiantes puedan entender el espacio geográfico que habitan.

En el caso chileno, las temáticas relacionadas con terremotos, tsunamis, aluviones, inundaciones y riesgos naturales se tratan en Historia, Geografía y Ciencias Sociales, en quinto y sexto año básicos, materias que son de análisis en esta investigación. En este sentido, diversos autores reconocen la falta de educación de la población chilena en esta temática, por ejemplo, Lagos, Cisternas y Mardones mencionan que:

"La construcción de viviendas sociales en áreas de riesgo de tsunami demuestra el desconocimiento y la falta de consideración de la recurrencia de eventos naturales extremos. Lamentablemente, Chile posee numerosos ejemplos de este hecho, evidenciando la incapacidad de aprender lecciones de eventos pasados. Solo han transcurrido casi cinco décadas del tsunami de 1960 y ya se han olvidado sus destructores efectos (2008: 13)".

En este contexto, es relevante tomar en cuenta las redes de apoyo para la educación que existen en las diversas organizaciones mundiales, puesto que trabajan en temáticas asociadas a la investigación y reducción de riesgos, en los cuales se destaca que, en muchos casos, la acción mundial concertada, en combinación con la educación de las personas, pueden contribuir a paliar el sufrimiento y a salvar vidas (UNICEF, 2019).

\section{Área de estudio}

El área de estudio se encuentra ubicada en la zona central-sur de Chile, correspondiente a la ciudad de Constitución en la costa de la Región del Maule (Figura $\mathrm{N}^{\circ} 1$ ). En términos demográficos, la localidad cuenta con 57.096 habitantes, de los cuales el 51\% corresponde a población masculina y el restante $(49 \%)$ a población femenina. Además, tiene 
un índice de ruralidad que alcanza al 19\%, fundamentalmente en localidades ubicadas en el sector norte de la comuna, en la costa sur y en las riberas del Río Maule, donde existen pequeños poblados que se mantienen asociados al funcionamiento del Ramal Talca-Constitución (Municipalidad de Constitución, 2016: 5).

Esta ciudad costera se desenvuelve dentro de áreas económicas diversas por su geografía, tales como "la actividad forestal, la pesca y la construcción, lo que atrae a personas de comunas aledañas a establecerse en busca de oportunidades laborales" (Municipalidad de Constitución, 2016: 6). Además, cabe destacar que los habitantes han posicionado la ciudad dentro de un emergente desarrollo de turismo en la región del Maule.

La ciudad de Constitución ha enfrentado desastres en los últimos años, tales como el terremoto y tsunami de 2010, incendios forestales en el verano de 2017 e inundaciones en 2018:

"El sábado 27 de febrero a las 03 horas y 34 minutos hora continental chilena, se produjo un terremoto. Su duración (3 minutos), su magnitud (8,8 grados Richter, la sexta mayor registrada en el mundo desde inicios del siglo XX) y la ubicación de su epicentro $\left(35,9^{\circ}\right.$ latitud sur y $72,7^{\circ}$ longitud oeste, 335 kilómetros al suroeste de Santiago, la zona que concentra más población en Chile) así lo confirman. Además, minutos después, el terremoto produjo un tsunami que azotó las costas de la zona central entre las regiones del Libertador Bernardo O'Higgins hasta la del Biobío, siendo especialmente destructivo en las costas de la Región del Maule. Dada la cantidad de víctimas y damnificados, fue esta última región la que recibió el mayor impacto de ambos eventos combinados. De hecho, en Constitución, principal ciudad costera de esta región, media hora después del terremoto, tres olas de 8,10 y 8 metros respectivamente, ingresaron por la desembocadura del río Maule y devastaron la ciudad" (Leiva-Bianchi, 2011: 552). 


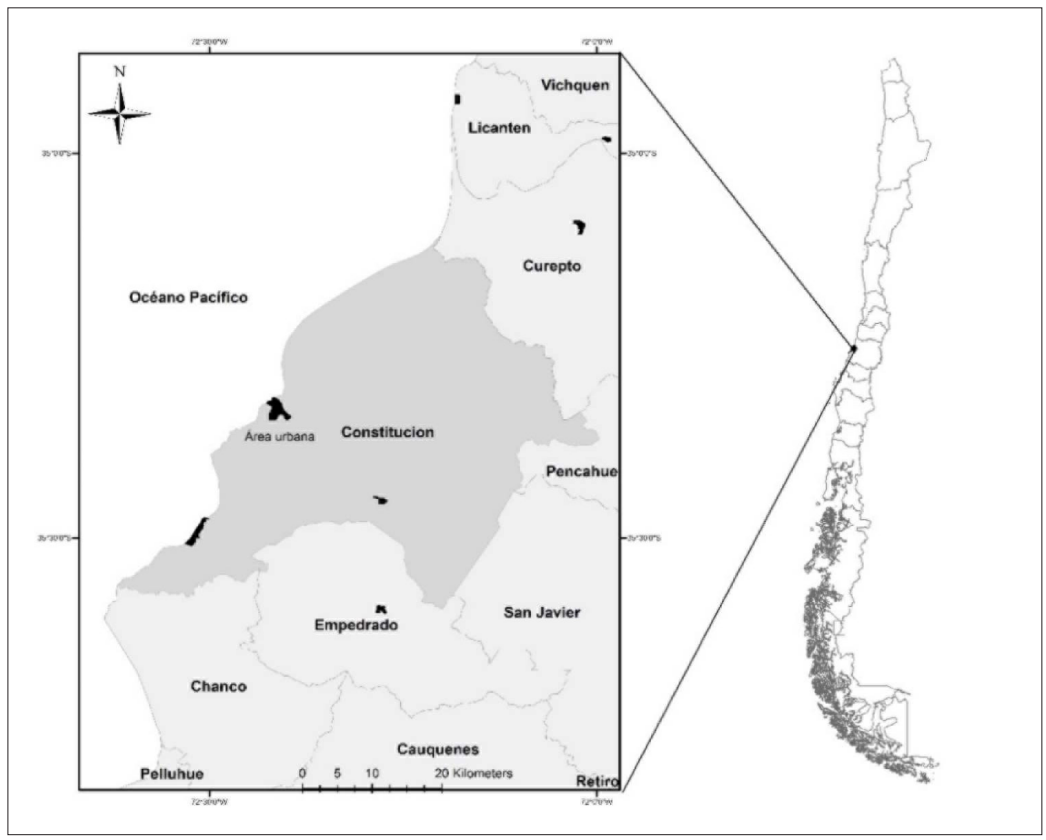

Figura $N^{\circ} 1$. Ubicación del área de estudio en Chile Fuente: Elaboración propia.

Sin embargo, en la educación formal en las escuelas del área de estudio, se desconoce qué grado de profundización existe en la materia, ya que, debido a la recurrencia de catástrofes en la zona, se puede tener la hipótesis que lo anterior ha fomentado una cultura en torno al conocimiento de la geografía y los riesgos naturales en las comunidades educativas. Por lo tanto, la problemática estará situada en verificar si los temas que se plantean en los planes y programas, en relación con los riesgos naturales y desastres, son aprendidos por los estudiantes, ya que lo anterior permitiría generar una cultura para reducir el riesgo de desastres en Constitución. Por lo anterior, se desprenden las siguientes preguntas: a) ¿qué contenidos geográficos se enseñan?; b) ¿cómo se enseñan los contenidos geográficos asociados a la temática de riesgos y desastres naturales? y c) los niños y niñas, ¿reflejan dominio de los contenidos geográficos asociados al currículo escolar en riesgos y desastres?

La presente investigación se llevó a cabo en cuatro establecimientos educacionales de carácter municipal de la comuna, ubicados en 
el área urbana de la ciudad, lo que corresponde al $100 \%$ de los establecimientos educacionales, tal como lo muestra la Figura № 2. Dos de estas instituciones están ubicadas a dos cuadras del Río Maule, una de las zonas más afectadas por el tsunami del 2010. En este caso, la Escuela Enrique Donn Müller quedó destruida por completo, por su infraestructura que data del siglo XX; la Escuela Nueva Bilbao no sufrió daños significativos, por su ubicación cercana a un territorio a mayor altura sobre el nivel del mar, pero aun así está en riesgo por su cercanía al Río Maule. Las otras dos escuelas están ubicadas en el sector alto de la ciudad, o cerros de la cordillera de la costa, altamente poblados; por un lado, Escuela José Opazo Díaz, emplazada en la zona de Cerro Alto o CORVI, y la Escuela Chacarillas, situada en la zona que lleva el mismo nombre, ambas cercanas a la entrada sureste de la ciudad. Estas últimas se encuentran en una zona catalogada como segura, por la altura del territorio, siendo beneficioso en caso de tsunami.

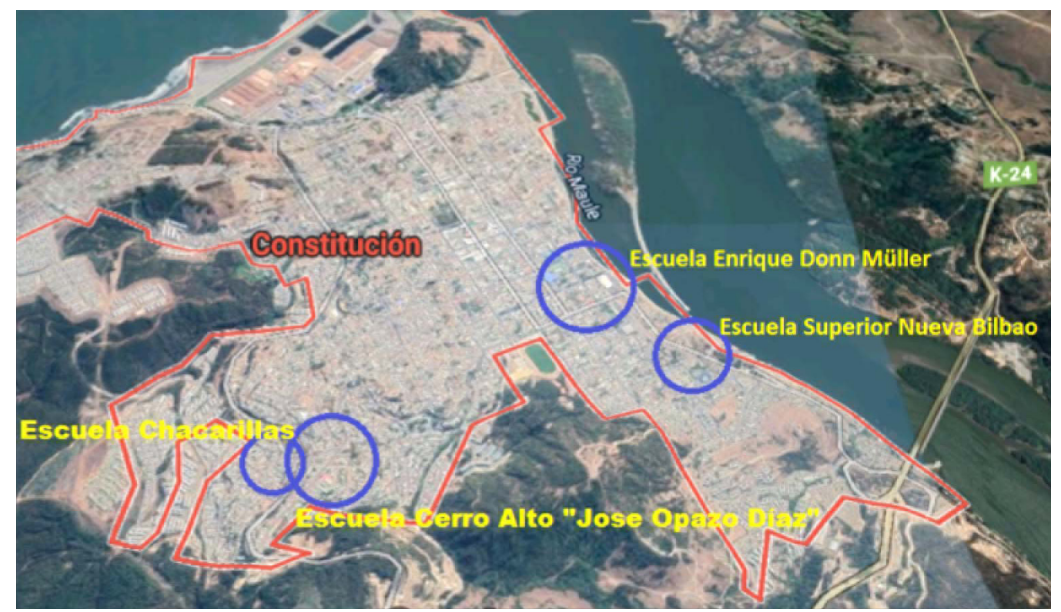

Figura $N^{\circ} 2$. Distribución de escuelas en el área urbana de Constitución Fuente: Google Earth.

\section{Metodología}

Se aplicó pruebas objetivas de contenido a un total de 216 alumnos, 106 de sexto y 110 de séptimo básico, de las escuelas Enrique Donn Müller, Nueva Bilbao, Cerro Alto José Opazo Díaz y Chacarillas. Además, cinco docentes fueron encuestados, entre ellos profesores básicos, especialis- 
tas, inspectores y jefes de las unidades técnico-pedagógicas (UTP), esto se debe a situaciones de reemplazo, licencias, ausencias o no contratación de docentes en el área de Historia, Geografía y Ciencias Sociales.

Los pasos secuenciales de la investigación se detallan en el flujo metodológico (figura No3). En este es posible observar el orden de la investigación; en primera instancia, el planteamiento del problema y su factibilidad de estudio, pasando luego a la generación de una hipótesis y objetivos. Posteriormente, se realizó la recopilación y revisión de bibliografía, resaltando el diseño de la investigación multimétodo, en la que se diseñaron instrumentos de recogida de información, como encuestas y evaluaciones de contenidos realizadas a los estudiantes; de esta forma se procesaron los datos y se obtuvieron los resultados. Finalmente, se consiguió un diagnóstico y una propuesta de diseño curricular orientada a la realidad local de la comuna de Constitución.

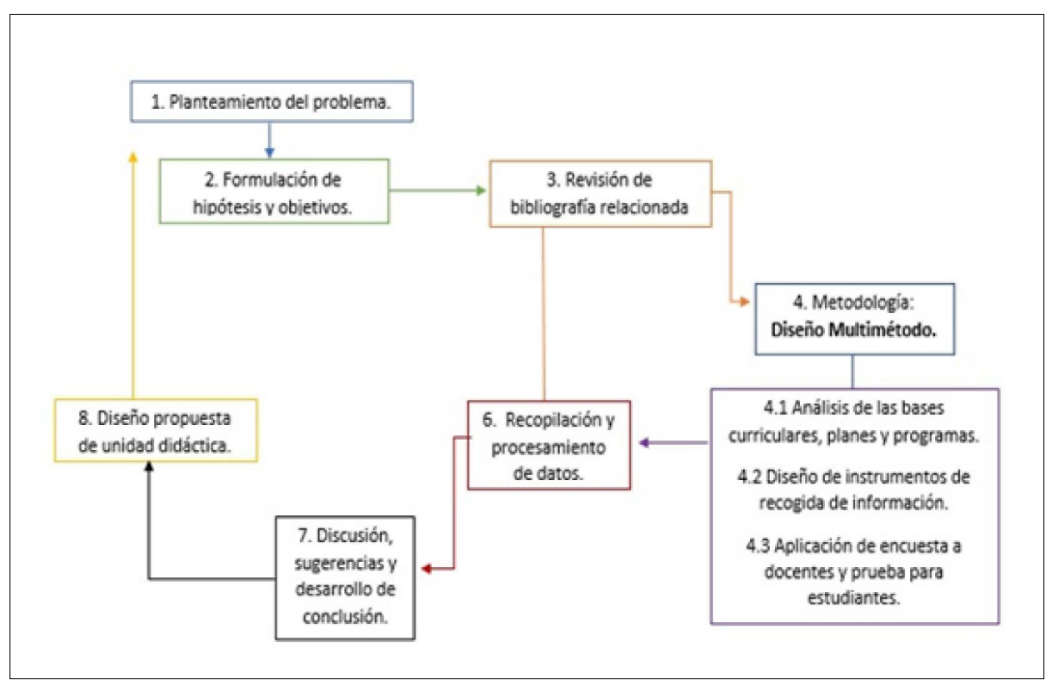

Figura $N^{\circ} 3$. Flujo metodológico

Fuente: Elaboración propia.

El diseño de investigación presenta características cuantitativas y cualitativas, metodología que algunos autores llaman "multimétodo". Fuenmayor (2017: 4) menciona que "este enfoque posee la implicación de un acopio de datos de diferente índole, tales como números, palabras, símbolos u otros, para someterse a análisis de interpretación, dis- 
cusión, teorización e incluso demostración si es el caso, a fin de construir y obtener conocimientos con la intención de cubrir de manera integral los diferentes ámbitos que envuelven el proceso investigativo". Con esto se busca cubrir la mayor cantidad de variables que influyen en el proceso de investigación, destacando además que, mediante el método cuantitativo, se logra un grado de objetividad de los resultados, sin generar alteraciones de los datos que se recogen. Así se logra justificar las conclusiones con datos medibles, sin la carga cultural que posean los o las investigadoras.

Este método se seleccionó por las características de la investigación, ya que se necesitan datos cuantitativos para dar respuestas asociadas a lo cualitativo, estableciendo una fusión de ambos para obtener mejores resultados, sin necesidad de enfocar a un solo método investigativo. Porque, "al perder de vista que la realidad no se corresponde en todo momento con un enfoque u otro, por sus limitaciones, al contrario, exige posibilitar la integración, por lo cual, al querer tener una visión de conjunto del fenómeno o problema, de sus diferentes aspectos y perspectivas, se hace necesaria la complementariedad multimetódica o mixta" (Fuenmayor, 2017:3).

Asimismo, Fuenmayor señala que:

"el docente investigador debe considerar que el análisis de resultados es transcendental en un estudio, pues de él depende el nivel de convicción y validez de dicha investigación, así como el conocimiento obtenido sobre el objeto de estudio; por tal razón, al utilizar el enfoque multimétodo propicia el acercamiento y comprensión del mismo en la confiabilidad, al igual que el estricto rigor con el cual fue estudiado, analizado, confrontado, y validado mediante la triangulación (2017: 22)".

Las técnicas de recogida de datos se pueden dividir en seis grandes grupos: observación, entrevistas, cuestionarios, pruebas objetivas y test, técnicas grupales y sociometrías, y análisis de documentos (Gil, 2016: 20). Se aplican en primer lugar pruebas objetivas, tipo test, sobre desastres y riesgos, a estudiantes de sexto y séptimo básico de establecimientos municipales, con el fin de evaluar su nivel de aprendizaje y manejo teórico conceptual en los niveles anteriores de escolaridad (quinto y sexto básico respectivamente). 
Además, se aplicó una encuesta a los docentes para obtener su percepción respecto de la enseñanza de riesgos y desastres. Se analizó cómo se enseña y el nivel de conocimientos que están incorporando los estudiantes acerca de la temática de desastres naturales y riesgos. Es necesario mencionar que el docente cumple un rol fundamental en la enseñanza de la geografía, por lo tanto es relevante medir su situación profesional como formador especialista del área de Historia, Geografía y Ciencias Sociales.

\section{Análisis de las bases curriculares, planes y programas de Historia, Geografía y Ciencias Sociales}

Para analizar la temática de riesgos, se trabajó sobre la base de identificar, en las bases curriculares, los contenidos asociados a la temática de riesgos y desastres naturales, a través de la revisión bibliográfica y consulta a la página web del Ministerio de Educación (www.mineduc.cl).

\section{Examen de las formas de enseñanza y manejo teórico conceptual que poseen los profesores de establecimientos municipales de la comuna de Constitución}

Para examinar las formas de enseñanza y manejo teórico conceptual, se realizó una encuesta a los docentes a cargo de los cursos en los que se tomaron las pruebas objetivas, con el objetivo de medir su nivel de conocimiento en relación con el contenido básico sobre desastres y riesgos naturales, tanto en el ámbito local como nacionalmente. En los cuatro establecimientos se realizaron cinco encuestas a docentes, completando el $100 \%$ de los establecimientos educacionales.

La encuesta se elaboró con respuestas cerradas, y el docente debía responder entre las opciones a) siempre, b) casi siempre, c) a veces y d) nunca. Además, se orientó a conocer el desempeño de los docentes, tanto en su labor de enseñanza como orientativa, centrándose en saber el nivel de importancia que da a los contenidos estudiados, el nivel de horas que dedica a la profundización de los contenidos y cómo los relaciona con los problemas locales y comunales del lugar donde se desempeña.

De igual forma, el diseño del instrumento de investigación se fundamentó en los lineamientos del marco para la buena enseñanza, se- 
leccionando ítems que tuvieran relación con los cuatro dominios que debe manejar un docente en el contexto escolar: Dominio A (preparación de la enseñanza), Dominio B (creación de un ambiente propicio para el aprendizaje), Dominio C (enseñanza para el aprendizaje de todos los estudiantes) y Dominio D (responsabilidades profesionales) (MINEDUC, 2008).

Se incorporaron también preguntas acerca de las exigencias básicas planteadas por el MINEDUC en sus bases curriculares, cuestionando cómo se enseñan los objetivos de aprendizaje que se exigen en la educación formal.

Evaluación del nivel de aprendizaje y manejo teórico conceptual de los estudiantes en relación con la temática de desastres naturales

Para evaluar el nivel de aprendizaje y el manejo conceptual de los estudiantes, se aplicaron dos pruebas de conocimientos, con objetivos extraídos directamente de los planes y programas del currículo nacional chileno y de los textos escolares que los estudiantes utilizan en su proceso educativo. Cabe destacar que las evaluaciones se hicieron para los niveles de sexto y séptimo básico del segundo ciclo educativo, con contenidos que fueron trabajados en el año anterior. Los estudiantes que rindieron las pruebas fueron 216 entre sexto y séptimo básico de todos los establecimientos, donde 106 son de sexto y 110 de séptimo.

Las pruebas se orientaron por áreas del conocimiento, diferenciándose para cada nivel educativo según los siguientes contenidos (Tabla $\left.\mathrm{N}^{\circ} 1\right)$. 
Tabla $\mathrm{N}^{\circ} 1$

Contenidos medidos en las evaluaciones

\begin{tabular}{|c|c|}
\hline Contenido o ítem & Desarrollo del ítem \\
\hline $\begin{array}{l}\text { Riesgos y desastres } \\
\text { naturales }\end{array}$ & $\begin{array}{l}\text { Este ítem se encuentra plasmado en nueve } \\
\text { preguntas dentro de la prueba ( } 6 \text { en sexto y } 3 \\
\text { en séptimo), enfocadas en los contenidos de } \\
\text { riesgos y desastres naturales que ocurren a } \\
\text { nivel nacional, buscando cubrir la totalidad } \\
\text { del país, es decir, desde las zonas naturales del } \\
\text { norte grande, norte chico, zona central, zona } \\
\text { sur, y austral de Chile. También, es importante } \\
\text { destacar que a preguntas de séptimo básico se } \\
\text { les asigna un enfoque local. }\end{array}$ \\
\hline Geografía física & $\begin{array}{l}\text { Para sexto y séptimo básico, enfocado en } \\
\text { resaltar las características geológicas de } \\
\text { la superficie terrestre, desde el relieve, } \\
\text { hidrografía, clima, vegetación, fauna, suelo, } \\
\text { etc. }\end{array}$ \\
\hline Conceptos geográficos & $\begin{array}{l}\text { Aplicado solo a sexto, destacando las nociones } \\
\text { sobre los principales riesgos y desastres } \\
\text { naturales que ocurren y afectan, tanto como } \\
\text { a nivel nacional como local. Es decir, evaluar } \\
\text { los conocimientos en cuanto al significado de } \\
\text { algunos riesgos y desastres, como temporales, } \\
\text { aluviones, o qué es un riesgo, etc. }\end{array}$ \\
\hline Prevención de riesgo & $\begin{array}{l}\text { Aplicada solo a sexto, se encuentra enfocada } \\
\text { en temas de prevención de riesgos naturales, } \\
\text { y ceñida en los contenidos que se encuentran } \\
\text { en el texto escolar de sexto básico y los planes } \\
\text { y programas. }\end{array}$ \\
\hline $\begin{array}{l}\text { Sistemas e instrumentos } \\
\text { de medición }\end{array}$ & $\begin{array}{l}\text { Aplicada solo a séptimo, también enfocada } \\
\text { en hacer relevancia a uno de los principales } \\
\text { sistemas de medición en materia de riesgos y } \\
\text { desastres sismográficos. }\end{array}$ \\
\hline $\begin{array}{l}\text { Instituciones } \\
\text { gubernamentales de } \\
\text { emergencia de Chile }\end{array}$ & $\begin{array}{l}\text { Aplicada solo a séptimo, busca dar un enfoque } \\
\text { primordial al funcionamiento y significado de } \\
\text { la ONEMI en Chile. }\end{array}$ \\
\hline
\end{tabular}

Fuente: Elaboración propia. 


\section{Resultados}

La geografía y el riesgo de desastres en el currículo nacional (bases curriculares, planes y programas)

Como resultado, tras el análisis y lectura del currículo nacional, se identificó que los contenidos referidos a geografía y riesgos naturales se desarrollan en quinto y sexto básico. La Tabla $\mathrm{N}^{\circ} 2$ muestra las unidades en que se trata la temática, el propósito y el número de horas pedagógicas; como se aprecia, se tiene un total de 56 horas pedagógicas, durante 12 años de educación formal.

El currículo propone solo dos objetivos de aprendizaje (OA), que se sintetizan en las tablas ㅇ3 y No4. Como se observa, el mismo plan de estudio sugiere a los docentes las formas de evaluar, desglosadas en "indicadores de evaluación sugeridos", por lo que el profesor, según su criterio, escogerá de qué forma abordar y evaluar los objetivos. Lo anterior es bastante subjetivo y la profundización de los temas va a depender muchas veces del interés del docente y de su capacitación en la materia.

En lo que se refiere a $5^{\circ}$ básico, el Objetivo de Aprendizaje (OA 12) señala: "Investigar, describir y ubicar los riesgos naturales que afectan a su localidad, como sismos, maremotos, inundaciones, derrumbes y volcanismo, e identificar formas en que la comunidad puede protegerse (construcciones antisísmicas, medidas de seguridad y evacuación en el hogar, en la escuela y en los distintos espacios públicos, entre otros)" (MINEDUC, 2013: 61).

Para el nivel $6^{\circ}$ básico, el objetivo de aprendizaje (OA) se refiere a: "Explicar cómo han influido los desastres naturales en el desarrollo de Chile durante su historia reciente, dando ejemplos a nivel nacional y regional (sismos, volcanismo, sequía, inundaciones y derrumbes, entre otros)" (MINEDUC, 2013: 166).

\section{La geografía, el riesgo de desastres y los docentes}

La Figura No5 corresponde a los resultados obtenidos en la encuesta realizada a los cinco docentes de los establecimientos municipales en la ciudad de Constitución, cumpliendo con el $100 \%$ de todos los establecimientos estudiados. Los profesores debían dar respuesta a diez preguntas. 
A partir de esta encuesta, se puede observar que no solo declaran cumplir con lo requerido por los planes y programas de la asignatura al momento de entregar los contenidos, sino que también buscan generar aprendizajes significativos por medio de la profundización en la temática de riesgos y desastres naturales. Esta profundización se refleja en las didácticas llevabas a cabo por los docentes, que permiten generar modificaciones curriculares focalizadas en esta temática.

Para lograr esta profundización, los docentes dan por hecho la necesidad de mantenerse actualizados frente a la temática de riesgos y desastres naturales, permitiéndose así mejorar su didáctica y dominio de los contenidos. Sin embargo, manifiestan que los contenidos entregados por el MINEDUC no son suficientes para formar ciudadanos preparados e íntegros, que logren enfrentar de forma pertinente una situación de riesgo o un desastre. Esto último es relevante, ya que muestra una deficiencia del sistema escolar chileno, esta carencia es reconocida por los docentes y es urgente de mejorar ya que cada vez los contenidos geográficos tienden a disminuir con el tiempo en la malla curricular de la educación formal

\section{Geografía física, riesgos y desastres naturales en los estudiantes de $6^{\circ}$ y $7^{\circ}$ año básico}

La prueba aplicada a un total de 216 estudiantes de los cuatro establecimientos educacionales urbanos de $6^{\circ}$ y $7^{\circ}$ año de enseñanza básica, arrojó un nivel bajo de aprobación y en todos los casos menor al 51\% (Figura №4). Así como la investigación evidenció bajas tasas de aprobación, a la inversa presentó altos niveles de desaprobación, lo que quiere decir que en algunos establecimientos educacionales alcanzó el $79 \%$.

De esta forma, se logra corroborar el bajo nivel de conocimiento en la materia y aprendizaje de los contenidos de los programas, demostrando también la necesidad de un programa de educación para la gestión de riesgos de desastres a nivel escolar, ya que los contenidos planteados en las bases curriculares, planes y programas del MINEDUC no demuestran ser suficientes en el presente caso. Además, como mencionan Lagos, Cisternas y Mardones (2018: 13), en Chile existe una incapacidad de aprender lecciones de eventos catastróficos anteriores. 


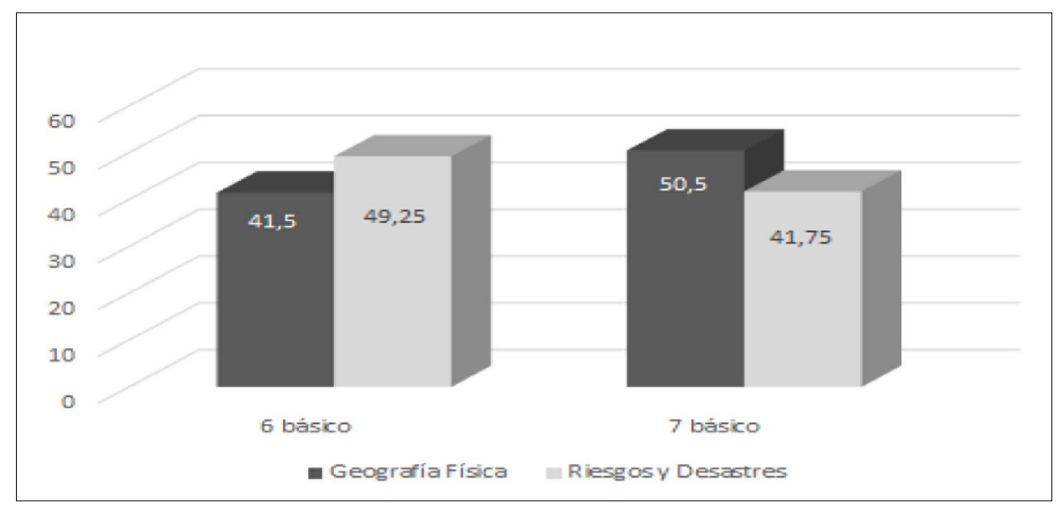

Figura $N^{\circ} 4$. Niveles de aprobación por curso y temáticas

Fuente: Elaboración propia.

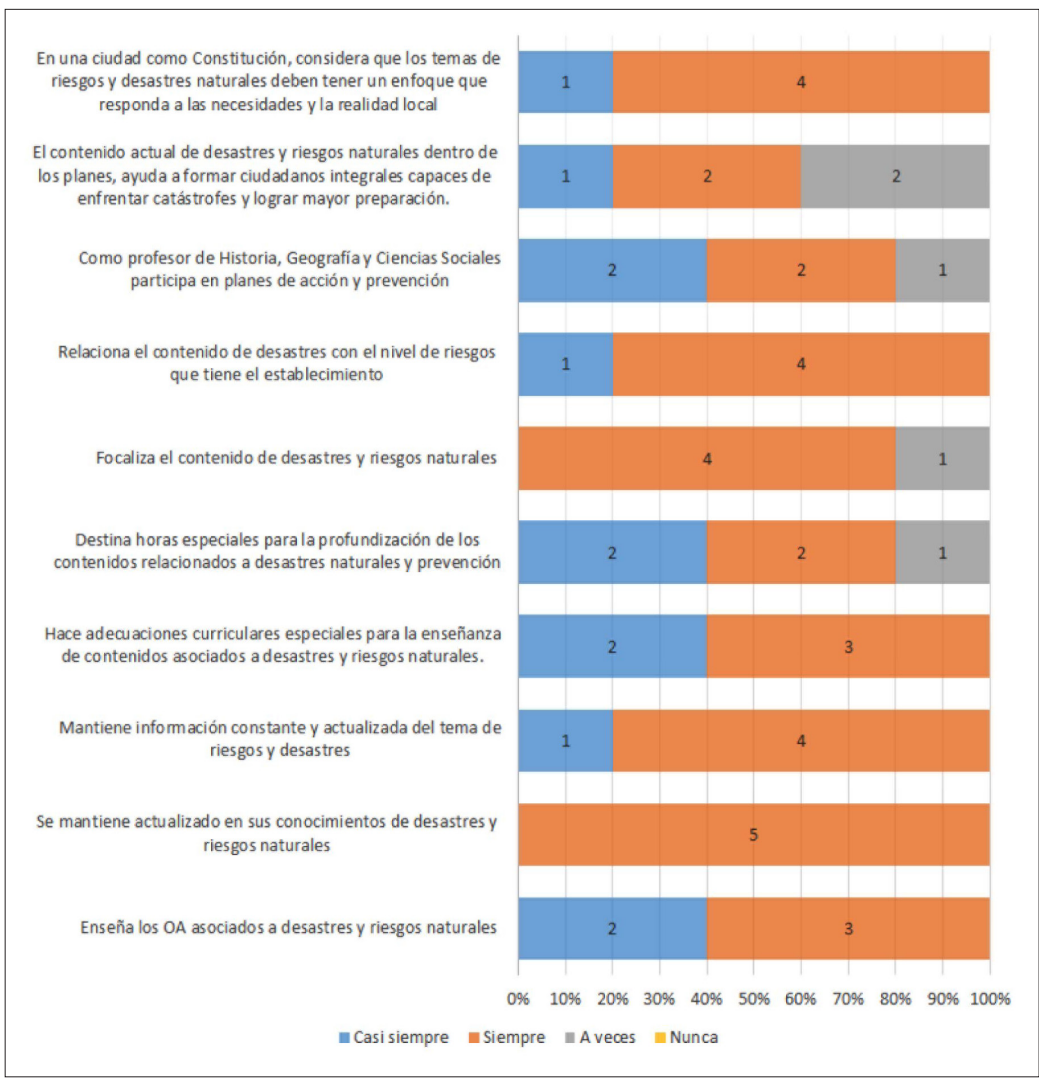

Figura $N^{\circ} 5$. Encuesta realizada a profesores

Fuente: Elaboración propia. 
Tabla №2

Características de las unidades de aprendizaje, propósito y horas pedagógicas

\begin{tabular}{|c|c|c|c|}
\hline Nivel & Unidad & Propósito & Horas \\
\hline $\begin{array}{l}\text { Quinto } \\
\text { año básico }\end{array}$ & $\begin{array}{l}\mathrm{N}^{\circ} 1 \\
\text { "La diversidad } \\
\text { geográfica de } \\
\text { Chile" }\end{array}$ & $\begin{array}{l}\text { "que los estudiantes tomen concien- } \\
\text { cia de la diversidad geográfica de } \\
\text { Chile y sean capaces de describir el } \\
\text { espacio geográfico chileno, conside- } \\
\text { rando los principales rasgos físicos } \\
\text { de las distintas zonas naturales del } \\
\text { país, los paisajes resultantes de la in- } \\
\text { terrelación del medio natural con la } \\
\text { sociedad y los principales recursos } \\
\text { presentes en cada una de estas zo- } \\
\text { nas" (MINEDUC, 2013: 58). }\end{array}$ & 32 \\
\hline $\begin{array}{l}\text { Sexto año } \\
\text { básico }\end{array}$ & $\begin{array}{l}\mathrm{N}^{\circ} 4 \\
\text { "Chile desde } \\
\text { las diversas } \\
\text { visiones } \\
\text { geográficas" }\end{array}$ & $\begin{array}{l}\text { "que los estudiantes conozcan las } \\
\text { características de nuestro territorio } \\
\text { desde cuatro perspectivas distintas. } \\
\text { En primer lugar, el reconocimiento } \\
\text { de elementos constitutivos del te- } \\
\text { rritorio nacional, relevantes tanto } \\
\text { desde el punto de vista geográfico, } \\
\text { en segundo lugar, está la organiza- } \\
\text { ción político-administrativa de Chi- } \\
\text { le, en tercer lugar, se encuentran los } \\
\text { diversos ambientes naturales exis- } \\
\text { tentes en nuestro país y finalmente, } \\
\text { se espera que los estudiantes tomen } \\
\text { conciencia de los desastres natura- } \\
\text { les que han afectado a Chile, a nivel } \\
\text { nacional y regional, a lo largo de su } \\
\text { historia" (MINEDUC, 2013: 160) }\end{array}$ & 24 \\
\hline
\end{tabular}

Fuente: Elaboración propia. 
Tabla N ${ }^{\circ} 3$

Habilidades, actitudes e indicadores del objetivo de aprendizaje (12), nivel $5^{\circ}$ básico

\begin{tabular}{|c|c|c|}
\hline Habilidades & Actitudes & $\begin{array}{c}\text { Indicadores de } \\
\text { evaluación sugeridos }\end{array}$ \\
\hline $\begin{array}{l}\text { *Usar herramientas } \\
\text { geográficas para ubi- } \\
\text { car, caracterizar y re- } \\
\text { lacionar elementos } \\
\text { del espacio geográfico, } \\
\text { como regiones, climas, } \\
\text { paisajes, población, re- } \\
\text { cursos y riesgos natura- } \\
\text { les (OA d). }\end{array}$ & $\begin{array}{l}\text { *Establecer lazos de } \\
\text { pertenencia con su en- } \\
\text { torno social y natural a } \\
\text { partir del conocimiento, } \\
\text { valoración y reflexión } \\
\text { sobre su historia perso- } \\
\text { nal, de su comunidad y } \\
\text { del país. }\end{array}$ & $\begin{array}{l}\text { *Obtienen información } \\
\text { de diversas fuentes } \\
\text { sobre los riesgos natu- } \\
\text { rales que afectan a su } \\
\text { localidad. } \\
{ }^{*} \text { Construyen un mapa } \\
\text { temático sobre los } \\
\text { principales focos de } \\
\text { riesgo de su localidad. }\end{array}$ \\
\hline $\begin{array}{l}\text { *Orientarse en el es- } \\
\text { pacio, utilizando ca- } \\
\text { tegorías de ubicación } \\
\text { absoluta (coordenadas } \\
\text { geográficas) y relativa } \\
\text { (OA e). }\end{array}$ & $\begin{array}{l}\text { tancia y la dignidad de } \\
\text { todos los trabajos, va- } \\
\text { lorando y respetando } \\
\text { a las personas que los } \\
\text { realizan. } \\
{ }^{*} \text { Comportarse y actuar }\end{array}$ & $\begin{array}{l}\text { *Definen un tema o } \\
\text { problema de investi- } \\
\text { gación sobre los prin- } \\
\text { cipales riesgos natu- } \\
\text { rales presentes en su } \\
\text { localidad (ej., carencia }\end{array}$ \\
\hline $\begin{array}{l}\text { *Formular y responder } \\
\text { preguntas para profun- } \\
\text { dizar sobre temas de su } \\
\text { interés, en relación con } \\
\text { el pasado, el presente o }\end{array}$ & $\begin{array}{l}\text { en la vida cotidiana se- } \\
\text { gún principios y virtu- } \\
\text { des ciudadanas }\end{array}$ & $\begin{array}{l}\text { de vías de evacuación, } \\
\text { creación de zonas se- } \\
\text { guras, identificación de } \\
\text { problemas naturales, } \\
\text { etc.). }\end{array}$ \\
\hline $\begin{array}{l}\text { el entorno geográfico } \\
\text { (OA h). }\end{array}$ & & $\begin{array}{l}\text { *Describen cuáles de } \\
\text { los riesgos que afectan }\end{array}$ \\
\hline $\begin{array}{l}\text { *Fundamentar opinio- } \\
\text { nes respecto de temas } \\
\text { estudiados en el nivel, } \\
\text { utilizando fuentes, da- } \\
\text { tos y evidencia (OA i). }\end{array}$ & & $\begin{array}{l}\text { a su localidad son co- } \\
\text { munes a la zona natu- } \\
\text { ral en que se ubica o a } \\
\text { otras zonas naturales } \\
\text { del país. }\end{array}$ \\
\hline $\begin{array}{l}{ }^{*} \text { Comparar distintos } \\
\text { puntos de vista respec- } \\
\text { to de un mismo tema } \\
\text { (OA j). }\end{array}$ & & $\begin{array}{l}\text { *Utilizan diversos re- } \\
\text { cursos gráficos (mapas, } \\
\text { imágenes, dibujos) } \\
\text { para exponer, de ma- } \\
\text { nera escrita, oral o vi- } \\
\text { sual, el tema indagado } \\
\text { y evaluar formas o me- } \\
\text { didas que ayudan a que } \\
\text { la población se proteja } \\
\text { de los fenómenos físi- } \\
\text { cos. }\end{array}$ \\
\hline
\end{tabular}

Fuente: Elaboración propia. 
Tabla $\mathrm{N}^{\circ} 4$

Habilidades, actitudes e indicadores del objetivo de aprendizaje (14), nivel $6^{\circ}$ básico

Habilidades

Actitudes
Indicadores de evaluación sugeridos
*Usar herramientas geográficas para ubicar, caracterizar y relacionar elementos del espacio geográfico, como regiones, climas, paisajes, población, recursos y riesgos naturales (OA d).

*Investigar sobre temas del nivel y aplicar distintas estrategias para registrar y organizarla información obtenida de dos o más fuentes sobre un tema (como organizadores gráficos, tablas, lista de ideas principales y esquemas, entre otros) (OA f).

*Formular y responder preguntas para profundizar sobre temas de su interés, con relación al pasado, al presente o al entorno geográfico (OA h).

*Fundamentar opiniones frente a temas estudiados en el nivel, utilizando fuentes, datos y evidencia (OA i).

*Evaluar posibles soluciones frente a un problema o proyecto y justificar su elección (OA k).
*Respetar y defender la igualdad de derechos esenciales de todas las personas, sin distinción de sexo, edad, condición física, etnia, religión o situación económica.

*Trabajar en forma rigurosa y perseverante, con espíritu emprendedor y con una disposición positiva a la crítica y la autocrítica.

*Establecer lazos de pertenencia con su entorno social y natural a partir del conocimiento, valoración y reflexión sobre su historia personal, de su comunidad y del país.
*Construyen una línea de tiempo u otra representación gráfica para ubicar temporalmente los principales desastres naturales que han afectado a Chile en el último siglo.

*Localizan en mapas las zonas de Chile donde estos desastres han tenido lugar (ej., erupciones de ciertos volcanes, lugares donde han ocurrido los mayores terremotos o las grandes inundaciones, entre otros).

*Construyen un mapa temático sobre los principales focos de riesgo que ha tenido y tiene el país.

*Explican las consecuencias que pueden tener para las personas algunos desastres naturales (por ejemplo, desplazamiento de población, daño en las construcciones e infraestructura, anegamiento, pérdida de cosechas y animales, entre otros).

*Proponen medidas concretas para prevenir las consecuencias de los desastres naturales.

*Investigan, utilizando diversas fuentes, acerca de los principales riesgos naturales presentes en su región.

*Utilizan diversos recursos gráficos (mapas, imágenes, dibujos) para exponer el tema indagado y evaluar formas o medidas que ayudan a la población a resguardarse de peligros naturales.

Fuente: Elaboración propia. 
Dominio de conceptos geográficos en las pruebas de conocimientos aplicados en $6^{\circ}$ básico

La aplicación de las pruebas demuestra a su vez bajos niveles de aprobación en el dominio de conocimientos acerca de conceptos geográficos relacionados con los principales riesgos y desastres naturales a nivel nacional. Por otro lado, en la sección "prevención de riesgos", que hace referencia a los contenidos de los textos escolares en que los estudiantes deberían estar familiarizados, presenta menos de un $40 \%$ de aprobación (Figura $\mathrm{N}^{\circ}$ 6). Lo anterior es preocupante; la familiarización de los estudiantes con la temática de prevención de riesgos debería de ser un compromiso social en una zona como Constitución, porque esto permitiría salvar vidas en el futuro.

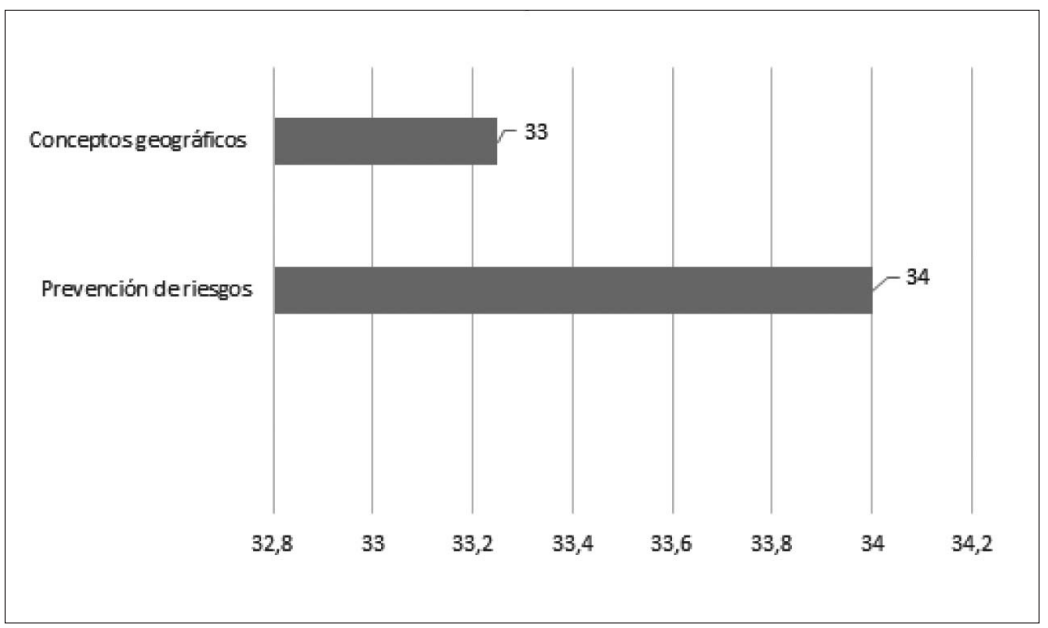

Figura $N^{\circ} 6$. Porcentaje de alumnos aprobados en $6^{\circ}$ básico Fuente: Elaboración propia.

Dominio de conceptos geográficos en las pruebas de conocimientos aplicados en $7^{\circ}$ básico

Con respecto al número de estudiantes que en este nivel aprobaron en las pruebas de conocimientos relacionados con los sistemas de medición en geografía y las instituciones gubernamentales de emergencia en Chile, los resultados fueron más favorables (Figura №7). Lo anterior es interesante de investigar, ya que en estos temas es necesario identificar el aporte de la educación informal al conocimiento, así como 
también la influencia de los medios de comunicación que pudieran estar influyendo en el grado de información.

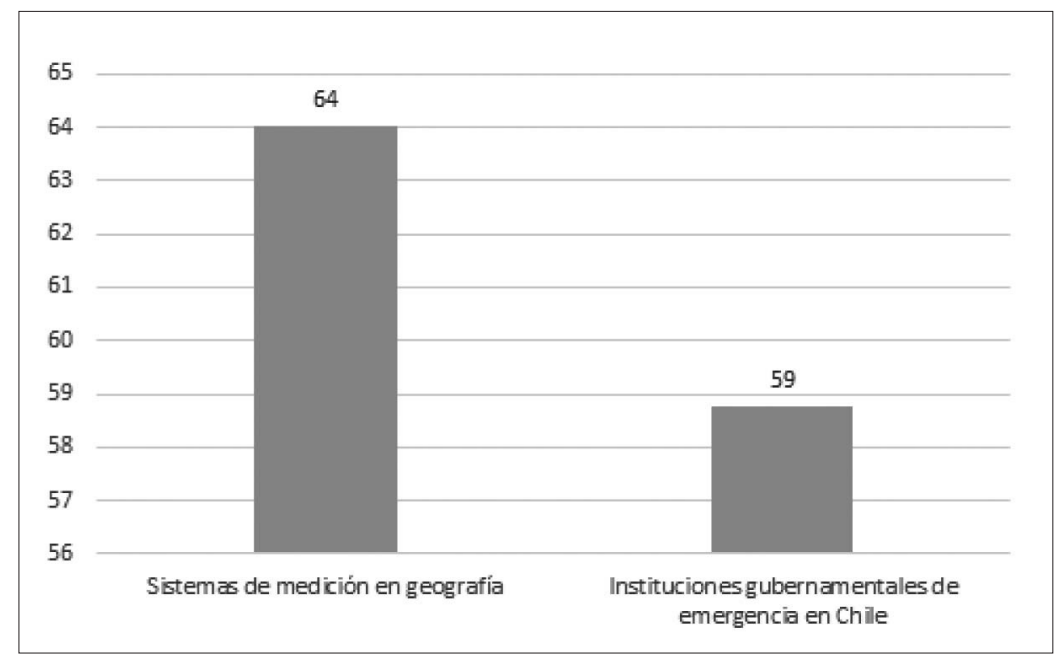

Figura $N^{o 7}$. Porcentaje de alumnos aprobados en $7^{\circ}$ básico Fuente: Elaboración propia.

\section{Resultados generales}

Al analizar los resultados generales de todos los ítems analizados, independientemente del nivel, se obtiene que aquellos en los que se presenta mayor tasa de desaprobación son los relacionados con conceptos básicos de geografía general, tales como conocimientos en cuanto al significado de algunos riesgos y desastres, como temporales, aluviones o qué es un riesgo, mientras que los que presentan mayor nivel de aprobación son los relacionados con sistemas e instrumentos de medición en geografía. En este sentido, sería interesante analizar las estrategias de enseñanza para abordar las distintas temáticas, ya que los docentes declaran estar innovando en ellas, lo que puede estar relacionado con los resultados de aprobación y desaprobación. 


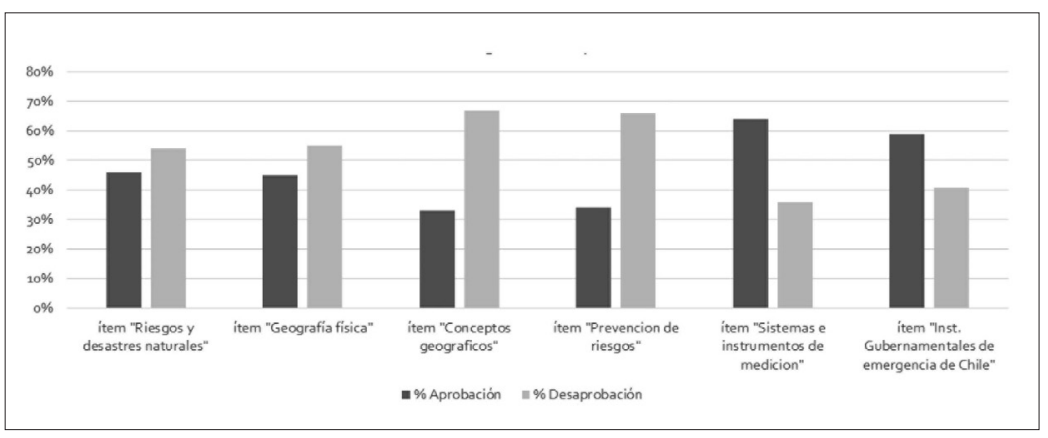

Figura $N^{\circ} 8$. Resultados generales de aprobación o desaprobación Fuente: Elaboración propia.

\section{Discusión}

Con el pasar de los años se han forjado nuevos modelos curriculares y, a partir de estos y de las reformas vigentes en educación, se entiende que la educación debe estar destinada a enfocarse permanentemente en las necesidades del estudiante, dejando menor participación al contenido, contexto y docente (Sandholtz, 1997). Si bien esto abre paso a una educación más amplia dentro del aula, existen materias que, debido a la orientación que posee la formación educacional, carecen de un enfoque permanente en el currículo nacional. Un ejemplo es la correspondiente a Historia, la cual, como asignatura, se divide en tres áreas disciplinares, Historia, Geografía y Ciencias Sociales. Dentro de estas ciencias, el área de la geografía pierde importancia en el contexto educacional nacional, lo que resulta contradictorio con el contexto geográfico e histórico de Chile (Arenas Martija, 2013: 143-162). La escasa relevancia que se le da a la disciplina geográfica trae por lo tanto consecuencias negativas al país, ya que, por las características del territorio nacional, la falta de alfabetización geográfica tiene como resultado una sociedad poco informada que, en zonas amenazadas por fenómenos naturales (terremotos, tsunamis, sequías e inundaciones) como es Constitución, aumenta el riesgo de desastres.

En el campo de la educación, la geografía ha sido desplazada por materias que poseen mayor preponderancia que esta rama (MINEDUC, 2012: 1), hecho reflejado en la cantidad de horas pedagógicas destinadas en al año, siendo ejemplo de esto las asignaturas de ma- 
temáticas, lenguaje y ciencias naturales. Esto se debe a que todas las disciplinas encasilladas en Historia carecen de estrategias de enseñanza y procedimientos de evaluación que hagan de la geografía una asignatura preponderante en el contexto educacional de Chile (Downs, 1994). Lo anterior queda demostrado en esta investigación, ya que, al revisar el currículo, se obtiene que en doce años de educación formal la temática de riesgos naturales y desastres solamente aparece en dos unidades.

Es importante considerar el saber espacial informal como un antecedente de prácticas transformativas en el discurso pedagógico (Garrido, 2005: 137), ya que, por medio de estos saberes, se puede innovar en didácticas curriculares que incluyan las experiencias de los sujetos con el espacio en el cual se desenvuelven. Por lo anterior, es importante conocer qué estrategias pedagógicas presentan mejores resultados en el aprendizaje de las temáticas relacionadas con riesgos y desastres. En esta investigación, por ejemplo, los docentes declararon innovar en sus estrategias, aspecto que sería interesante contrastar con las pruebas que presentaron mejores porcentajes de aprobación.

La escasa importancia que se da a geografía a nivel educacional es preocupante, debido a que el país no está exento de enfrentar desastres naturales. En esta realidad, los desastres naturales generan no solo consecuencias monetarias, sino también en el desempeño educacional de los alumnos, debido a la interrupción abrupta de su aprendizaje por resultado de un repentino desastre que pueda presentarse (Bassi, 2010).

Existe consenso internacional en que la educación es una herramienta eficaz para reducir el riesgo de desastres. En 2006 la Oficina de las Naciones Unidas para la Reducción del Riesgo de Desastres, UNISDR, UNESCO y UNICEF lanzaron la campaña internacional “La prevención de desastres empieza en la escuela”, que consideró determinante avanzar en la Prioridad 3 del Marco de Acción de Hyogo: "Utilizar el conocimiento, la innovación y la educación para crear una cultura de seguridad y resiliencia a todo nivel" (Khamis y Osorio, 2012).

Ahora bien, los resultados de la encuesta a los docentes en Constitución son categóricos en destacar que los contenidos en materia 
de riesgos y desastres no son suficientes, que los profesores necesitan mantenerse actualizados en los contenidos y mejorar sus prácticas pedagógicas. De acuerdo con lo anterior, hay mucho que hacer respecto de entregar herramientas que permitan a los docentes mejorar los aprendizajes, lo que también requiere de un esfuerzo por parte del país para cumplir con acuerdos internacionales. Por ejemplo, en 2011 Chile firmó la declaración de Panamá, la cual, en uno de los acuerdos, considera: "Fomentar la incorporación y/o el fortalecimiento de la gestión del riesgo en el currículum y el desarrollo de competencias docentes, en todos los niveles y modalidades educativas, de acuerdo con las políticas específicas de cada país" (Declaración de Panamá, 2011).

\section{Conclusiones}

La educación geográfica y el riesgo de desastres están fuertemente relacionados, ya que, en la medida en que la población posea una cultura acerca del riesgo de desastres, el riesgo disminuye. Por lo cual, en el escenario actual de la ciudad de Constitución, en la que los docentes encuentran insuficientes los contenidos y los estudiantes no poseen dominio de conceptos geográficos claves, urge fortalecer la educación geográfica considerándola una tarea pendiente.

Los docentes de las escuelas de Constitución enseñan básicamente los contenidos que aparecen en las bases curriculares, planes y programas; sin embargo, mencionan que estos son insuficientes y que es necesaria mayor capacitación para innovar en estrategias de aprendizaje que permitan mejorar su labor.

En las escuelas del área de estudio, existe un bajo grado de profundización en temáticas y contenidos geográficos relacionados con los riesgos y desastres naturales, por lo que la recurrencia de eventos catastróficos no ha fomentado una cultura relacionada con el conocimiento de estos tópicos en dichas comunidades educativas. Los resultados no demuestran una relación entre la frecuencia de desastres documentados a través de los años - especialmente después del tsunami y terremoto del 2010-, con los conocimientos de la comunidad educativa en los niveles investigados. Lo anterior es preocupante, por lo que esta investigación levanta la necesidad de realizar un programa de educación para la gestión de desastres a nivel escolar, para la preven- 
ción. En este sentido, es relevante que los profesores apliquen métodos de observación del medio, de análisis, historia local y geográfica, aprovechando al máximo los lugares transitados por los estudiantes, dando un valor identitario a lo que aprenden en el aula.

Al revisar el currículo nacional se puede constatar el reducido o limitado énfasis de los contenidos de geografía en la educación formal chilena, ya que, por ejemplo, un tema fundamental, como lo es la educación para el riesgo de desastres, se revisa solamente en $5^{\circ}$ y $6^{\circ}$ de enseñanza básica. Los resultados de esta investigación también arrojan bajos niveles generales de aprobación en conocimientos acerca de aspectos fundamentales del entorno geográfico, desencadenando una serie incongruencias entre las características geográficas de nuestro país, con los contenidos y la profundización que se entregan en las aulas. Entendiendo que Chile es un país expuesto a diversos fenómenos de origen natural, en los planes y programas no se evidencia una preocupación por la preparar a los estudiantes ante emergencias: Se debe considerar que estas temáticas se trabajan únicamente en dos niveles durante toda la escolaridad, restándole importancia social en un país con una diversidad de "riesgos asociados a", como es Chile.

\section{Referencias bibliográficas}

Fuentes primarias

a) Publicaciones oficiales

Chile. Ministerio de Educación. (2008). Marco para la Buena Enseñanza. Recuperado de https://www.docentemas.cl/docs/ MBE2008.pdf, revisado el 23-IV-2019.

Chile. Ministerio de Educación. (2012). Aprueba planes y programas de estudio de Educación Básica. Santiago de Chile: Biblioteca del Congreso Nacional de Chile.

Chile. Ministerio de Educación. (2013). Historia, Geografía y Ciencias sociales, programa de estudio Quinto año Básico. Santiago de Chile: Ministerio de Educación.

Chile. Ministerio de Educación. (2013). Historia, Geografía y Ciencias sociales, programa de estudio Sexto año Básico. Santiago de Chile: Ministerio de Educación. 
Chile. Ministerio de Educación. (2013). Curriculum Nacional. Recuperado de https://www.curriculumnacional.cl/614/articles-18972_programa.pdf, revisado el 23-IV-2019.

Declaración de Panamá (2011). Conferencia Internacional para la Reducción del Riesgo de Desastres en el Sector Educativo en América Latina y el Caribe, 12 al 14 de octubre de 2011. Recuperado de https://eird.org/pr14/cd/documentos/espanol/AmericadelSurHerramientasydocumentos/Informesregionalynacionales/doc19240-contenido.pdf, revisado el 23-IV-2019.

Khamis, M. y C. Osorio (2012). Análisis de riesgos de desastres en Chile. UNESCO.

Municipalidad de Constitución (2016). Actualización plan de desarrollo comunal. Constitución: Municipio de Constitución.

Naciones Unidas. (2007). Tomando Acciones con un Propósito en Común. Actas de la primera sesión de la Plataforma Global para la Reducción del Riesgo de Desastres. Ginebra, del 5 al 7 de junio del 2007. Recuperado de https://www.preventionweb.net/files/1464_PlataformaGlobal2007.pdf, revisado el 23-IV-2019.

UNESCO. (2019). Educación para la gestión del riesgo ante desastres. Recuperado de http://www.unesco.org/new/es/santiago/ education/disaster-risk-management-education/?fbclid=Iw AR1XJuIEA0pynh20L3TICER34YSgUeBkAOKhN0QWFXoT2FQlI8PkcBOn6oA, revisado el 23-IV-2019.

UNISDR (2009). Terminología sobre Reducción del Riesgo de Desastres. Recuperado de http://www.unisdr.org/files/7817_UNISDRTerminologySpanish.pdf, revisado el 23-IV-2019.

Fuentes secundarias

a) Artículos y capitulos de libros

Alderman, D. (2002). "Humor and film in the geography classroom: Learning from Michael Moore's TV Nation", en Journal of Geography, vol. 101, № 6, pp. 228- 239.

Arenas Martija, A. (2013). “Giros en la Educación Geográfica; renovación de lo geográfico y lo educativo", en Revista de Geografía Norte Grande, $\mathrm{N}^{\circ} 56$, pp. 143-162. 
Bednarz, R. S. (2004). "Geography education: The glass is half full and it's getting fuller", en The Professional Geographer, vol. 56, $\mathrm{N}^{\circ} 1$, pp. 22-27.

Briones-Gamboa, F. (2007). "La complejidad del riesgo: breve análisis transversal", en Revista de la Universidad Cristóbal Colón, vol. 20, No 3, pp. 9-19.

Downs, R. M. (1994). “The need for research in geography education: It would be nice to have some data", en Journal of Geography, vol. 93, $\mathrm{N}^{\circ} 1$, pp. 57-60.

ISDR (2011). "La gestión del riesgo en el ámbito educativo", en Revista EIRD Informa-Las Américas. Recuperado de https://www. eird.org/esp/revista/no_17_2010/art14.html, revisado el 23IV-2019.

Fuenmayor, R. y O. M. Bittar (2017). “Multimétodo. Visión paradigmática integradora en la investigación educativa”, en CICAG: Revista del Centro de Investigación de Ciencias Administrativas $y$ Gerenciales, vol. 15, N¹, pp. 211-225.

Garrido, M. (2005). “El espacio por aprender, el mismo que enseñar: las urgencias de la educación geográfica”, en Cad. CEDES, vol. 25, $\mathrm{N}^{\circ} 66$, pp. 137-163.

Leiva-Bianchi, M. (2011). "Relevancia y prevalencia del estrés posttraumático post-terremoto como problema de salud pública en Constitución", en Revista de Salud Pública, vol. 13, № 4, pp. 551-559.

Lagos, M., M. Cisternas y M. Mardones (2008). “Construcción de Viviendas Sociales en Áreas de Riesgo de Tsunami”, en Journal of Construction. Revista de la Construcción.

Molina, F., J. Constanzo y C. Inostroza, (2018). “Desastres naturales y territorialidad: el caso de los lafkenche de Saavedra”, en Revista de Geografía Norte Grande, N 71, pp. 189-209.

Ordóñez-Díaz, M., L. Montes-Arias y G. Garzón-Cortes (2017). “Importancia de la educación ambiental en la gestión del riesgo socio-natural en cinco países de América Latina y el Caribe”, en Revista Electrónica Educare, vol. 22, $\mathrm{N}^{\circ} 1$.

Reyes Rivero, A., A. Montilla Pacheco, P. Castillo García y M. Zambrano Vera (2017). "Amenaza, vulnerabilidad y riesgo ante eventos naturales. Factores socialmente construidos", en Journal of Science and Research. Revista Ciencia e Investigación, vol. 1, No6, pp. 22-28. 
b) Libros

Alpízar, M. L. (2009). Educación y Reducción de Riesgos y Desastres en Centroamérica: Gestión del Riesgo. San José, Costa Rica: Colección Pedagógica Formación Inicial de Docentes Centroamericanos de Educación Primaria o Básica.

Bassi, M. y S. Urzúa (2010). Educación en Chile: El desafío está en la calidad. Santiago de Chile: Banco Interamericano de Desarrollo.

Camus P., F. Arenas y F. Mendoza (2017). Conceptos y herramientas prácticas para un Manual de Reducción de Riesgo de Desastre en Chile. Recuperado de http://geografia.uc.cl/images/destacados/MANUAL_FINAL_nov.pdf, revisado el 23-IV-2019.

Gil, J. A. (2016). Técnicas e instrumentos para la recogida de información. Madrid: Universidad Nacional de Educación a Distancia.

Petit-Breuilh Sepúlveda, M. E. (2004). Desastres naturales y ocupación del territorio en Hispanoamérica. España: Universidad de Huelva.

Sandholtz, J. H. (1997). Teaching whit technology: Creating student-centered classrooms. New York: Columbia University. 